\title{
Ursolic Acid Causes Cell Death in PC-12 Cells by Inducing Apoptosis and Impairing Autophagy
}

\author{
JUNEYOUNG JUNG ${ }^{1}$, JEONGBIN SEO ${ }^{1}$, JOUNGMOK KIM ${ }^{2}$ and JEONG HEE KIM ${ }^{1,2}$ \\ ${ }^{1}$ Department of Life and Nanopharmaceutical Sciences, Graduate School, and \\ ${ }^{2}$ Department of Oral Biochemistry and Molecular Biology, Kyung Hee University, Seoul, Republic of Korea
}

\begin{abstract}
Ursolic acid (UA) is a natural pentacyclic triterpene that has various biological activities, including anticancer and anti-inflammatory effects. This study investigated the ability of UA to cause cell death in pheochromocytoma (PC-12) cells. UA was cytotoxic to $P C-12$ cells (half-maximum inhibitory concentration $=53.2$ $\mu M)$ and significantly reduced the clonogenic ability of $P C$ 12 cells. It also triggered apoptosis by reducing the level of B-cell lymphoma 2 (BCL2), activating caspase-3, and inducing cleavage of poly (ADP-ribosyl) polymerase. To investigate the effects of UA treatment on the induction and progression of autophagy, the levels of p62 and the conversion of the microtubule-associated protein light chain 3 (LC3)-I to LC3-II, which are important markers of autophagic flux, were monitored. UA treatment induced the accumulation of p62 and increased the LC3-II/LC3-I ratio. These results demonstrate that UA treatment induced autophagy, but the downstream signaling pathway was blocked. In summary, this study shows that UA kills PC-12 cells by inducing apoptosis and impairing autophagy progression.
\end{abstract}

Ursolic acid (3- $\beta$-hydroxy-urs-12-en-28-oic acid; UA) (Figure 1 ) is a natural pentacyclic triterpene that can be isolated from various plants, apple peel, and many other fruits and herbs used in daily life (1). UA has various biological and pharmacological effects, including anticancer activities against breast cancer (2), cervical cancer, osteosarcoma (3, 4), and prostate cancer (5), anti-inflammatory activity (6), anti-atherosclerosis activity (7), and antiviral activity (8).

Apoptosis is a selective physiological process that plays a critical role in balancing cell proliferation and cell death. A

Correspondence to: Professor Jeong Hee Kim, Department of Biochemistry and Molecular Biology, School of Dentistry, Kyung Hee University, Seoul 02447, Republic of Korea. Tel: +82 29610915, Fax: +82 29601467, e-mail: jhkimh@khu.ac.kr

Key Words: Ursolic acid, apoptosis, autophagy, PC-12 cell. wide range of stimuli can be integrated to trigger the irreversible decision for a cell to die. Many cytotoxic and neurotoxic agents cause cell death via apoptosis $(9,10)$. Autophagy is essential for cell survival and the maintenance of homeostasis. Recent studies indicate that autophagy also functions in cell death, and is called type II programmed cell death (11). Growing evidence suggests that crosstalk occurs between apoptosis and autophagy and that this helps to control cell survival and death $(12,13)$.

PC-12 is a rat pheochromocytoma cell line of neuroendocrine origin derived from the adrenal glands. This cell line has been used as a model system to evaluate the cytotoxic and neurotoxic effects of various substances (1416). We used PC-12 cells to elucidate the mechanism underlying the anticancer effects of UA.

\section{Materials and Methods}

Materials. The following chemicals were obtained from SigmaAldrich (St. Louis, MO, USA): UA, dimethyl sulfoxide (DMSO), Nonidet P (NP)-40, crystal violet powder, NaF, ethylenediaminetetra-acetic acid (EDTA), $\mathrm{Na}_{3} \mathrm{VO}_{4}$, and ethidium bromide. 3(4,5-dimethylthiazol-2-yl)-2,5-diphenyl-tetrazolium bromide (MTT) was purchased from Duchefa (Haarlem, the Netherlands). Ethanol, methanol, isopropanol, and isoamyl alcohol were obtained from Hayman Chemical Co. (Witham, Essex, UK), Merck Millipore (Darmstadit, Germany), and Junsei (Chou-ku, Japan), respectively. Bradford protein assay dye reagent was purchased from Bio-Rad (Hercules, CA, USA). A protease inhibitor was obtained from Roche Diagnostics (Indianapolis, IA, USA). Polyvinylidene difluoride (PVDF) membrane was purchased from Merck Millipore (Darmstadit, Germany). Cell culture materials were purchased from Welgene (Seoul, Korea) and Gibco BRL (Waltham, MA, USA). All other chemicals were of the highest analytical grade and purchased from common sources.

Rabbit polyclonal antibodies against B-cell lymphoma 2 (BCL2), caspase-3, human BCL2-associated X protein (BAX), poly (ADPribosyl) polymerase (PARP), LC3, and p62 were purchased from Cell Signaling Technology (Beverly, MA, USA). Mouse polyclonal antibodies against Beclin-1, $\alpha$-tubulin and vinculin were obtained from Santa Cruz Biotechnology (Dallas, TX, USA) and SigmaAldrich, respectively. Anti-mouse and anti-rabbit horseradish 


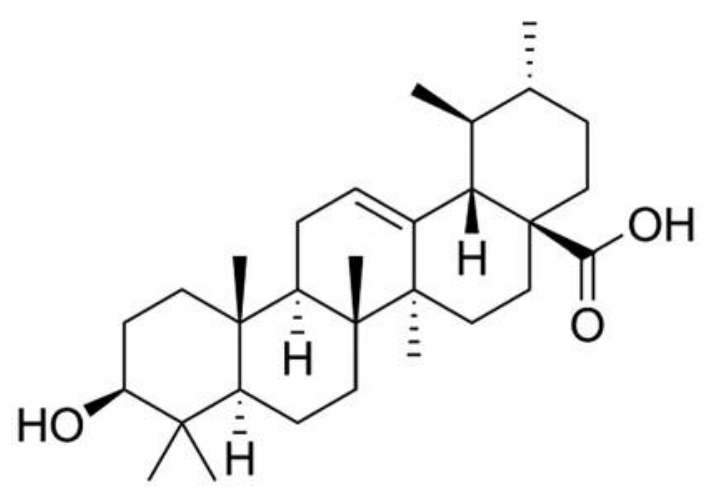

Figure 1. Structure of ursolic acid (3- $\beta$-3-hydroxy-urs-12-ene-28-oicacid).

peroxidase-conjugated secondary IgG antibodies were from Bethyl (Montgomery, TX, USA). West Pico Chemiluminescent Substrate was purchased from Thermo Scientific (Waltham, MA, USA).

Cell culture and UA treatment. Rat pheochromocytoma PC-12 cells (ATCC CRL-1721; ATCC, Manassas, VA, USA) were maintained in the logarithmic phase of growth in Dulbecco's modified Eagle's medium (DMEM; Welgene) supplemented with $5 \%$ fetal bovine serum (FBS; Gibco BRL), 5\% horse serum, $2 \mathrm{mM} \mathrm{L-glutamine,} \mathrm{and}$ antibiotics. Cultures were maintained at $37^{\circ} \mathrm{C}$ in a humidified atmosphere of $95 \%$ air and $5 \% \mathrm{CO}_{2}$. Logarithmically growing PC12 cells were used in all experiments. UA was dissolved in DMSO at a concentration of $20 \mathrm{mM}$ and diluted in tissue culture medium before use. For glucose starvation experiments, PC-12 cells were cultured in glucose-free DMEM (Welgene) supplemented with $10 \%$ FBS (Gibco BRL), $2 \mathrm{mM} \mathrm{L-glutamine,} \mathrm{and} \mathrm{antibiotics} \mathrm{for} \mathrm{0-24} \mathrm{h.}$

Cytotoxicity analysis. Cell viability was estimated by the MTT assay. Exponentially growing cells were seeded into a 96-well plate at a density of $3 \times 10^{4}$ cells/well and treated with different concentrations $(0-200 \mu \mathrm{M})$ of UA for $20 \mathrm{~h}$. Thereafter, $20 \mu \mathrm{l}$ of MTT $(5 \mathrm{mg} / \mathrm{ml})$ was added and the cells were incubated for another $4 \mathrm{~h}$ at $37^{\circ} \mathrm{C}$. The medium was carefully removed and $150 \mu \mathrm{l}$ of dissolving solvent $(4 \mathrm{mM} \mathrm{HCl}$ and $0.1 \% \mathrm{NP}-40$ prepared in isopropanol) was added. The plate was gently agitated until the blue formazan crystals were fully dissolved. Absorbance was measured at $550 \mathrm{~nm}$ using a microplate reader (Wallac Victor 3-V; Perkin Elmer, Waltham, MA, USA). The data are expressed as the mean percentage of viable cells in comparison with the control culture. All experiments were performed at least three times.

Colony-formation assay. Cells in log phase were seeded into a 6well plate at a density of $1 \times 10^{3}$ cells/well and treated with $0-50 \mu \mathrm{M}$ UA for $24 \mathrm{~h}$. Cells were then cultured in fresh medium for a further 14 days to allow colonies to form. Colonies were fixed with $100 \%$ methanol, stained with $0.5 \%$ crystal violet solution, and counted.

Western blot analysis. PC-12 cells were treated with different concentrations of UA $(0-50 \mu \mathrm{M})$ for $24 \mathrm{~h}$ and then lysed in lysis buffer (20 mM Tris, $100 \mathrm{mM} \mathrm{NaCl}, 0.1 \% \mathrm{NP}-40,50 \mathrm{mM} \mathrm{NaF}, 2$ $\mathrm{mM}$ EDTA, $1 \mathrm{mM} \mathrm{Na} \mathrm{VO}_{4}$, and a protease inhibitor, $\mathrm{pH}$ 7.5). The
A
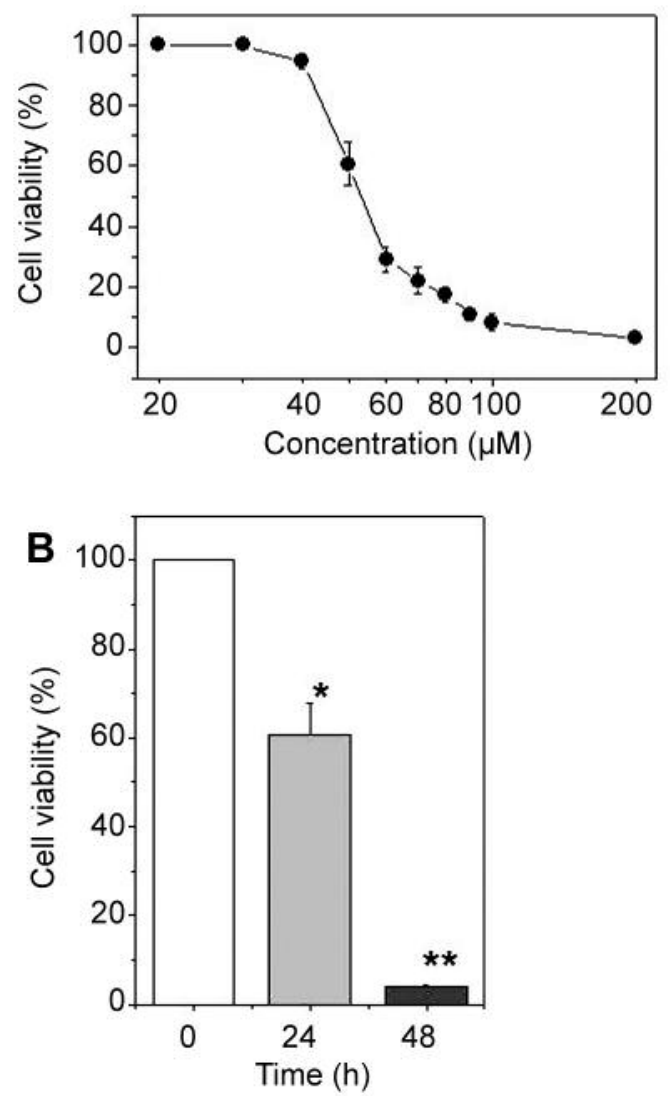

Figure 2. Cytotoxic effects of ursolic acid (UA) on PC-12 cells. A: Cells were treated with the indicated concentrations of UA for $24 \mathrm{~h}$, and then cell viability was measured by the 3-(4,5-dimethylthiazol-2-yl)-2,5diphenyl-tetrazolium bromide (MTT) assay. B: Cells were treated with $50 \mu M U A$ for 0,24 , and $48 \mathrm{~h}$, and then cell viability was measured by the MTT assay. Significantly different at $* p<0.01, * * p<0.001 \mathrm{vs}$. untreated control group. All experiments were performed at least in triplicate. Data are means \pm S.E.

protein concentrations of the lysates were determined by the Bradford assay. Alternatively, lysis buffer L (phosphate-buffered saline containing $1 \%$ Triton X-100 and $50 \mathrm{mM} \mathrm{NaF}$ ) was used to analyze autophagy-related proteins. Total protein (5 or $10 \mu \mathrm{g}$ of each lysate) was separated by sodium dodecyl sulfatepolyacrylamide gel electrophoresis and electro-transferred to PVDF membranes. The membranes were blocked with $5 \%$ non-fat milk for $1 \mathrm{~h}$ at room temperature and then probed with specific primary antibodies stated above for $16 \mathrm{~h}$ at $4^{\circ} \mathrm{C}$. The specific protein bands were visualized using a peroxidase-conjugated secondary antibody and chemiluminescent substrate solution. The data were quantified with Image $\mathbf{J}$ software and the relative ratio was calculated.

Statistical analysis. All data represent means \pm S.E. Statistical analysis was performed using analysis of variance of followed by the Student's $t$-test. 
A

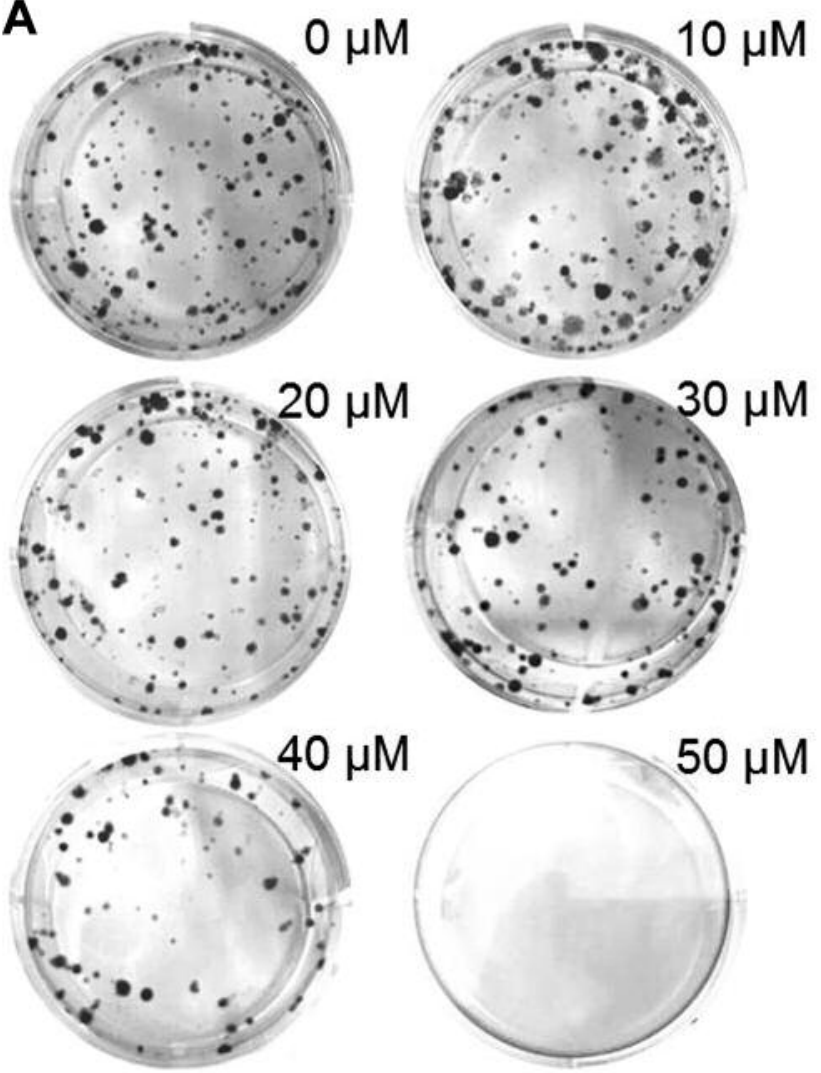

B

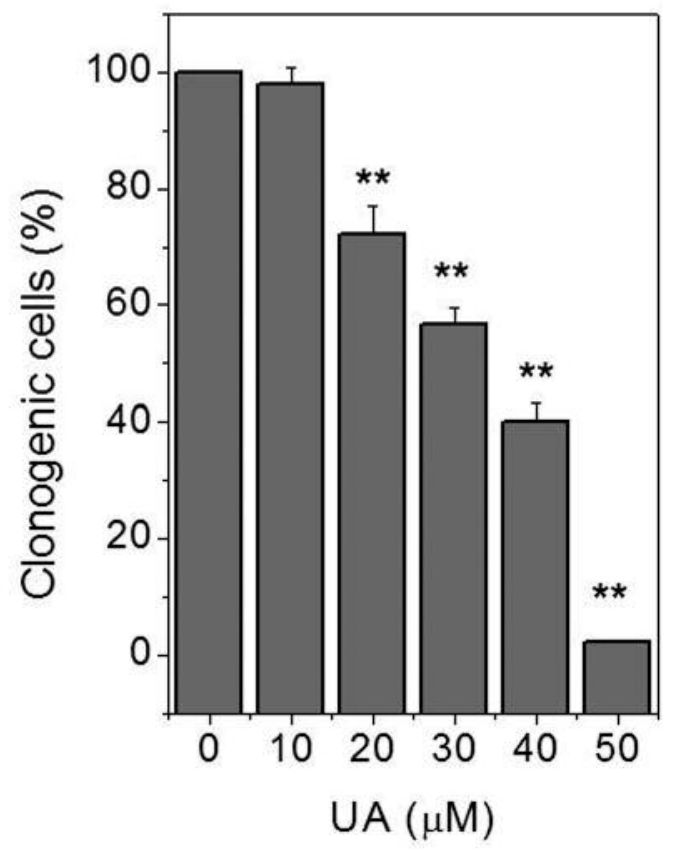

Figure 3. Effect of ursolic acid (UA) on colony formation by PC-12 cells. A: Cells were treated with different concentrations of UA for 24 h, incubated in fresh medium for a further 2 weeks, then fixed, and stained with crystal violet. B: The number of colonies containing more than 50 cells was counted. Relative percentages of clonogenic cells are plotted as means $\pm S D$ of three independent experiments. Significantly different at $* * p<0.001$ vs. untreated control group.

\section{Results}

$U A$ reduces the viability and proliferation of $P C-12$ cells. The effect of UA on cell survival was evaluated using the MTT assay. Treatment with UA for $24 \mathrm{~h}$ dramatically reduced the viability of PC-12 cells in a dose-dependent manner (Figure $2 \mathrm{~A})$. The concentration of UA required to inhibit viability by $50 \%\left(\mathrm{IC}_{50}\right)$ was approximately $53.2 \mu \mathrm{M}$. Cell viability was also determined after treatment with $50 \mu \mathrm{M}$ UA for different durations. Prolonged exposure to UA markedly reduced the viability of PC-12 cells (Figure $2 \mathrm{~B}$ ). The viability of control cells treated with vehicle alone was not affected.

The effect of UA on cell proliferation was examined by the colony-formation assay. After treatment as described in the Materials and Methods section, colonies containing more than 50 cells were counted. Consistent with the findings of the MTT assay, UA treatment greatly reduced the number of colonies compared with the number formed by untreated control cells (Figure 3). These data strongly suggest that UA markedly attenuates the growth of PC-12 cells.
UA induces apoptosis of PC-12 cells. To determine whether apoptosis is involved in the cytotoxic and antiproliferative effects of UA, we analyzed the expression levels of different apoptosis-related proteins. PC-12 cells were treated with $0-50 \mu \mathrm{M}$ UA for $24 \mathrm{~h}$, total proteins were isolated, and levels of BCL2, BAX, caspase-3, and PARP were measured by western blotting. This revealed that UA treatment reduced the protein level of BCL2 (Figure 4), an important regulator of apoptotic signaling pathways (17). The level of the pro-apoptotic protein BAX was not significantly affected. UA induced proteolytic processing of caspase-3 in a dose-dependent manner. Activation of caspase- 3 leads to the cleavage of a number of proteins, including PARP. Although PARP is not essential for cell death, its cleavage is a hallmark of apoptosis. UA treatment induced proteolytic cleavage of PARP in a dose-dependent manner, as exemplified by accumulation of the $89 \mathrm{kDa}$ cleaved form and disappearance of the full-length $116 \mathrm{kDa}$ PARP protein (Figure 4). 
A

$$
\begin{array}{lllll}
0 & 10 & 20 & 50 & (\mu \mathrm{M})
\end{array}
$$

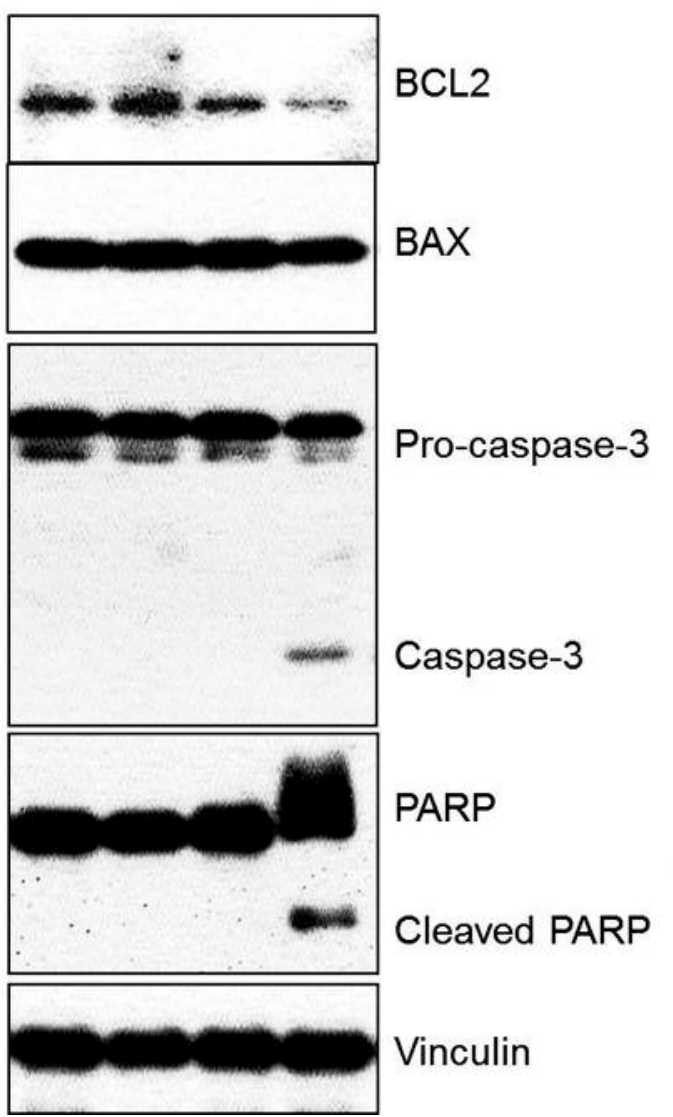

B
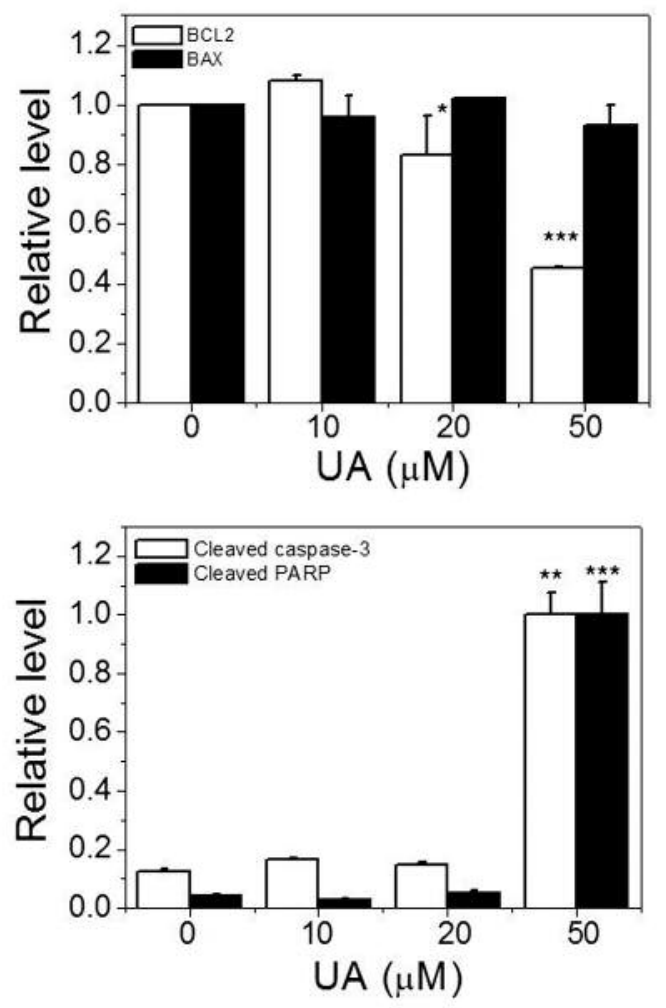

Figure 4. Effect of ursolic acid (UA) on expression of apoptosis-related proteins. $P C$ - 12 cells were treated with different concentrations of UA for 24 h. A: Cell extracts were subjected to western blotting to determine the levels of B-cell lymphoma 2 (BCL2), BCL2-associated X protein (BAX), caspase-3, and poly (ADP-ribosyl) polymerase (PARP). Representative western blots are shown. B: Relative intensities of BCL2 and BAX (upper panel), and activated caspase-3 and cleaved PARP (lower panel) bands are shown. Data are means \pm S.E. Significantly different at $* p<0.1,{ }^{* *} p<0.005$, *** $p<0.001$ vs. untreated control group.

UA impairs autophagy progression. We investigated whether UA induces an autophagy-related mechanism leading to type II programmed cell death in PC-12 cells. To confirm that PC-12 cells undergo autophagy under stressed conditions and to determine the optimal time points at which to observe changes in the levels of autophagy marker proteins, PC-12 cells were cultured in glucose-free medium for $0-24 \mathrm{~h}$. Under these conditions, the level of p62 decreased and the conversion of LC3-I to LC3-II increased in a time-dependent manner (Figure 5). These effects were particularly pronounced after glucose (Glc) starvation for $24 \mathrm{~h}$. However, the level of Beclin-1 was not changed under our experimental conditions.
UA treatment increased the conversion of LC3-I to LC3II in PC-12 cells in a dose-dependent manner (Figure 5). On the other hand, expression of p62 was increased, suggesting that it was accumulated, rather than degraded. The level of Beclin-1 was not significantly changed in UA-treated PC-12 cells.

\section{Discussion}

This study investigated the cytotoxic effect of UA on PC-12 cells. UA significantly reduced the survival of PC-12 cells, with an $\mathrm{IC}_{50}$ value of $53.2 \mu \mathrm{M}$. In addition, it significantly reduced the colony-formation ability of PC-12 cells. 
A

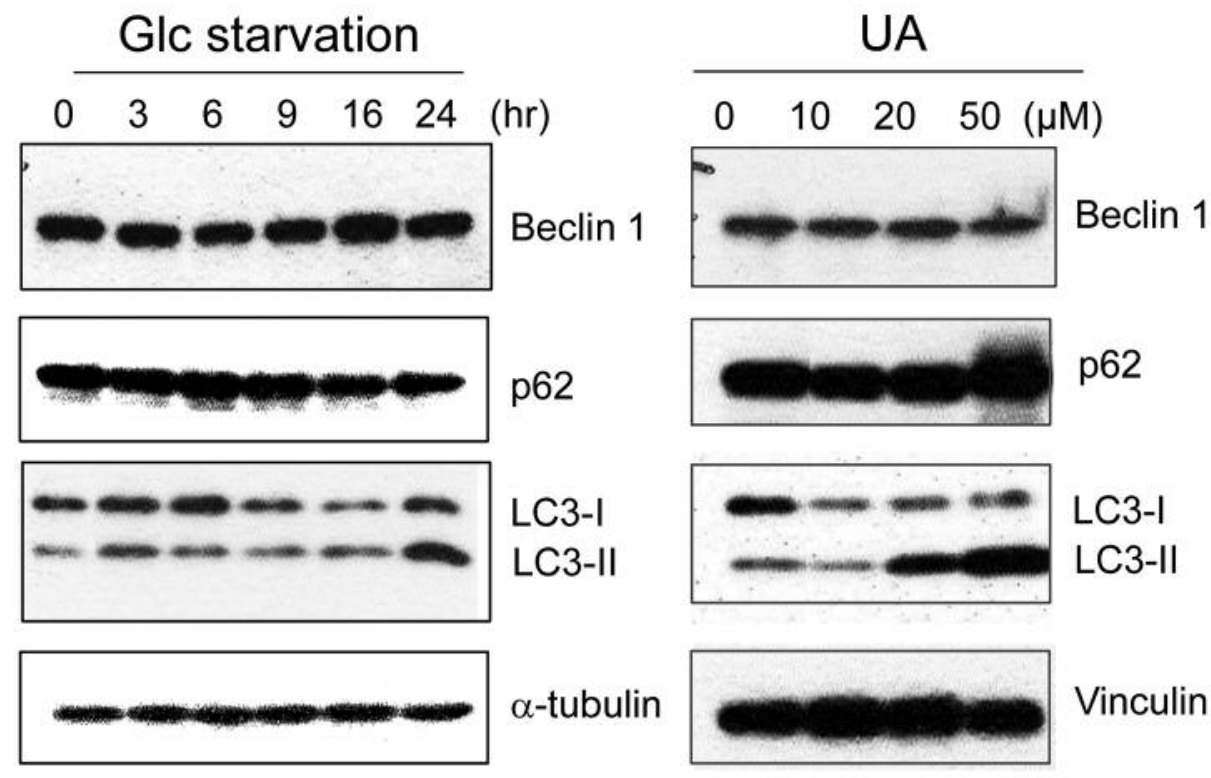

\section{B Glc starvation}
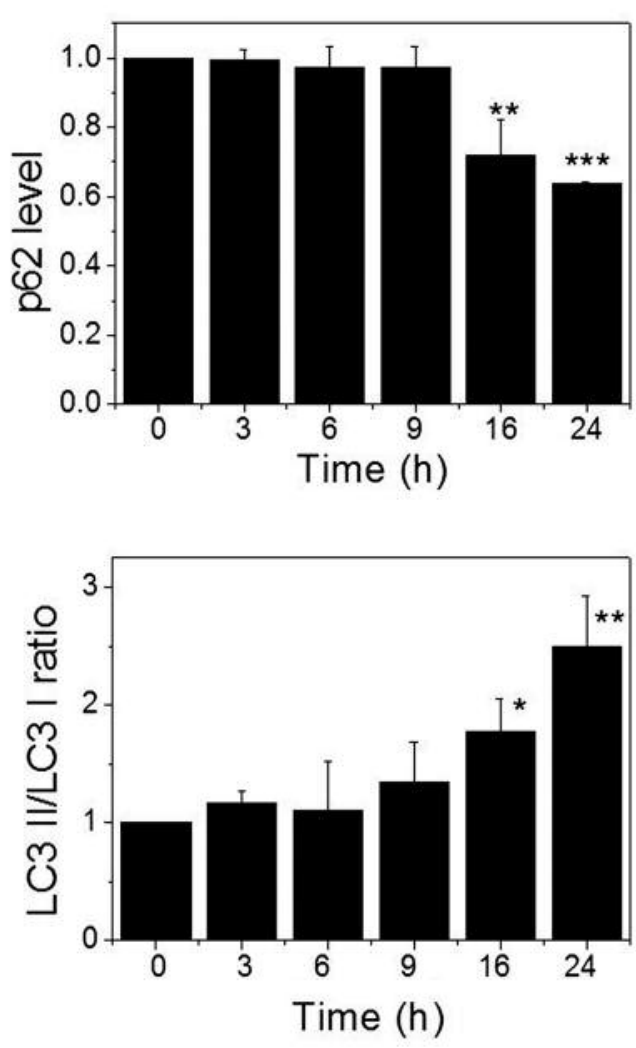

UA
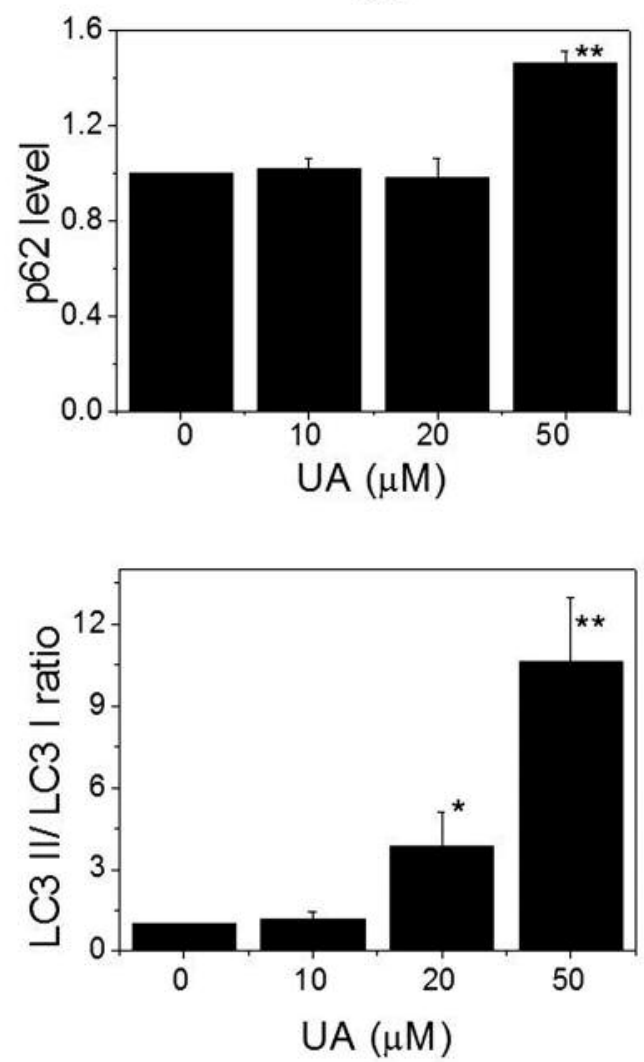

Figure 5. Effect of ursolic acid (UA) on expression of autophagy-related proteins. A: PC-12 cells were cultured in glucose (Glc)-free medium for the indicated duration to induce autophagy (left panel). Alternatively, cells were treated with different concentrations of UA for $24 \mathrm{~h}$ in complete medium (right panel). Changes in the levels of autophagy markers [beclin-1, p62, and the microtubule-associated protein light chain 3 (LC3)] were monitored. Representative western blots are shown. B: Relative intensity of the p62 band (upper panels) and the LC3-II/LC3-I ratio (lower panel) in glucosestarved and UA-treated cells are shown. Data are means \pm S.E. Significantly different at $* p<0.05, * * p<0.01, * * * p<0.001$ vs. untreated control group . 
The level of the anti-apoptotic protein BCL2 was reduced by UA, while the level of the pro-apoptotic protein BAX remained constant, resulting in a decreased BCL2/BAX ratio, one of the major events in regulating apoptosis (18). Caspase-3 was activated and the caspase substrate PARP was proteolytically cleaved in UA-treated PC-12 cells. Similarly, the level of BCL2 was reduced and PARP cleaved following caspase activation in UA-treated human cancer cells $(3,19,20)$.

Autophagy is an evolutionarily-conserved intracellular catabolic process that recycles macromolecules and mediates the lysosomal degradation of dysfunctional proteins, damaged organelles, and unnecessary cytoplasmic components $(21$, 22). This pathway is activated by metabolic stress conditions such as nutrient starvation, DNA damage, and organelle damage $(23,24)$. Accumulating evidence has revealed that autophagy is altered in various human diseases, including cancer. Autophagy can act both positively and negatively with regard to cancer cell survival $(21,25)$. Although autophagy serves as a protective mechanism by facilitating the degradation of superfluous or damaged cellular constituents, the blockade of this process prevents autophagosomelysosome fusion and thus this process does not progress further; impaired autophagy induces cell death (24).

One of the best characterized proteins in the autophagy pathway is p62, which interacts with LC3 and is subsequently incorporated into autophagosomes and degraded (26). The conversion of LC3-I to LC3-II is used as a marker of autophagosomes and to monitor autophagy (2729). As autophagy is induced and progresses, the level of p62 decreases and the conversion of LC3-I to LC3-II increases. These changes were observed in this study in glucose-starved PC-12 cells. UA treatment induced the accumulation of LC3II, indicative of an increased number of autophagosomes. However, there was no concomitant degradation of p62; rather, this protein accumulated. These data suggest that UA treatment inhibited autophagic flux in PC-12 cells.

It was recently reported that treatment with natural products can induce both apoptosis and autophagy in cancer cells $(30,31)$. Inhibition of autophagy enhanced resveratrolinduced apoptosis of esophageal squamous carcinoma cells (30). Blockade of autophagic flux enhanced apoptosis induced by the alkaloid matrine in human hepatoma cells (31). The natural compound oblongifolin $\mathrm{C}$ also inhibits autophagic flux by preventing autophagosome-lysosome fusion and autophagosome degradation and thus enhanced antitumor efficacy in human cervical carcinoma cells (25). In addition, metformin was found to promote apoptosis and autophagy in esophageal carcinoma cells (32). Similarly, we show here that UA induced apoptosis and autophagy in PC12 cells, but progression of autophagy was impaired.

In conclusion, we demonstrated that PC-12 cell death caused by UA is not induced by a single mechanism, but by a combination of two separate mechanisms; namely the induction of apoptosis and impaired autophagy. UA treatment inhibited proliferation and colony formation of PC-12 cells. Apoptosis was induced via reduction of expression of the antiapoptotic protein BCL2 and activation of caspase-3. The accumulation of p62 and increased conversion of LC3-I to LC3-II demonstrated that autophagy progression was impaired.

\section{Acknowledgements}

This study was supported by a grant from the BIO \& Medical Technology Development Program of the National Research Foundation of Korea (MEST, 2012M3A9C6049936).

\section{References}

1 Cargnin ST and Gnoatto SB: Ursolic acid form apple pomace and traditional plants: A valuable triterpenoid with functional properties. Food Chem 220: 477-489, 2017.

2 Lewinska A, Adamczyk-Grochala J, Kwaniewicz E, Deregowska A and Wunk M: Ursolic acid-mediated changes in glycolytic pathway promote cytotoxic autophagy and apoptosis in phenotypically different breast cancer cells. Apoptosis 22: 800815, 2017.

$3 \mathrm{Wu} \mathrm{CC}$, Chen $\mathrm{CH}$, Lee YH, Chang IL, Chen HY, Hsieh CP and Chueh PJ: Ursolic acid triggers apoptosis in human osteosarcoma cells via caspase activation and the ERK 1/2 MAPK pathway. J Agric Food Chem 64: 4220-4226, 2016.

4 Zhang RX, Li Y, Tian DD, Liu Y, Nian W, Zou X, Chen QZ, Zhou LY, Deng ZL and He BC: Ursolic acid inhibits proliferation and induces apoptosis by inactivating $\mathrm{Wnt} / \beta$ catenin signaling in human osteosarcoma cells. Int J Oncol 49: 1973-1982, 2016.

5 Gai WT, Yu DA, Wang XS and Wang PT: Anticancer effect of ursolic acid activates apoptosis through ROCK/PTEN-mediated mitochondrial translocation of cofilin-1 in prostate cancer. Oncol Lett 4: 2880-2885, 2016.

6 Kashyap D, Sharma A, Tuli HS, Punia S and Sharma AK: Ursolic acid and Oleanolic acid: Pentacyclic terpenoids with promising anti-inflammatory activities. Recent Pat Inflamm Allergy Drug Discov 10: 21-33, 2016.

7 Jiang Q, Hao R, Wang W, Gao $\mathrm{H}$ and Wang C: SIRT1/ATG5/autophagy are involved in the antiatherosclerosis effects of ursolic acid. Mol Cell Biochem 420: 171-184, 2016.

8 Zhao J, Chen J, Liu T, Fang J, Wan J, Zhao J, Li W, Li J, Zhao $\mathrm{X}$ and Chen S: Anti-viral effects of ursolic acid on guinea pig cytomegalovirus in vitro. J Huazhong Univ Sci Technolog Med Sci 32: 883-887, 2012.

9 Kwon K, Kwon YS, Kim SW, Yu K, Lee KH and Kwon OY: Luteolin-induced apoptosis through activation of endoplasmic reticulum stress sensors in pheochromocytoma cells. Mol Med Rep 16: 380-386, 2017.

10 Shailasree S, Venkataramana M, Niranjana SR and Prakash HS: cytotoxic effect of p-coumaric acid on neuroblastoma N2a cells via generation of reactive oxygen species leading to dysfunction of mitochondria inducing apoptosis and autophagy. Mol Neurobiol 51: 119-130, 2015.

11 Baehrecke EH: Autophagy: Dual roles in life and death? Nat Rev Mol Cell Biol 6: 505-510, 2005. 
12 Mukhtar E, Adhami VM, Khan N and Mukhtar H: Apoptosis and autophagy induction as mechanism of cancer prevention by naturally occurring dietary agents. Curr Drug Target 13: 1831$1841,2012$.

13 Mukhopadhyay S, Panda PK, Sinha N, Das DN and Bhutia SK: Autophagy and apoptosis: Where do they meet? Apoptosis 19: 555-566, 2014.

14 Tian X, Ru Q, Xiong Q, Yue K, Chen L, Ma B, Gan W, Si Y, $\mathrm{Xiao} \mathrm{H}$ and Li C: Neurotoxicity induced by methamphetamineheroin combination in PC12 cells. Neurosci Lett 647: 1-7, 2017.

15 Li B, Duan P, Han X, Yan W and Xing Y: NICD inhibits cell proliferation and promotes apoptosis and autophagy in PC12 cells. Mol Med Rep 16: 2755-2760, 2017.

16 Bullov P, Cougnoux A, Abunimer L, Kopacek J, Pastorekova S and Pacak K: Hypoxia potentiates the cytotoxic effect of piperlongumine in pheochromocytoma models. Ontarget 7: 40531-40545, 2016.

17 Reed JC: BCL-2 family proteins. Oncogene 17: 3225-3226, 1998.

18 Oltvai ZN and Korsmeyer SJ: Checkpoints of dueling dimers foil death wishes. Cell 79: 189-192, 1994.

19 Chuang WL, Lin PY, Lin HC and Chen YL: The apoptotic effect of ursolic acid on SK-HEP-1 cells is regulated by the PI3K/AKT, p38 and JNK MAPK signaling pathways. Molecules 21: 460, 2016.
$20 \mathrm{Li} \mathrm{Y,} \mathrm{Lu} \mathrm{X,} \mathrm{Qi} \mathrm{H,} \mathrm{Li} \mathrm{X,} \mathrm{Xiao} \mathrm{X} \mathrm{and} \mathrm{Gao} \mathrm{J:} \mathrm{Ursolic} \mathrm{acid} \mathrm{induces}$ apoptosis through mitochondrial intrinsic pathway and suppression of ERK1/2 MAPK in HeLa cells. J Pharmacol Sci 125: 202-210, 2014.

21 Yang $\mathrm{Z}$ and Klionsky DJ: Eaten alive: A history of macroautophagy. Nat cell Biol 12: 814-822, 2010.

22 Lin L and Baehrecke EH: Autophagy, cell death, and cancer. Mol Cell Oncol 2: e985913, 2015.

23 Levine B and Kroemer G: Autophagy in the pathogenesis of disease. Cell 132: 27-42, 2008.

24 Shimizu S, Yoshida T, Tsujioka M and Arakawa S: Autophagic cell death and cancer. Int J mol Sci 15: 3145-3153, 2014.

25 Lao Y, Wan G, Liu Z, Wang X, Ruan P, Xu W, Xu D, Xie W, Zhang $\mathrm{Y}, \mathrm{Xu} \mathrm{H}$ and $\mathrm{Xu} \mathrm{N}$ : The natural compound oblongifolin $\mathrm{C}$ inhibits autophagic flux and enhances antitumor efficacy of nutrient deprivation. Autophagy 10: 736-749, 2014.

26 Mizushima N and Komatsu M: Autophagy: renovation of cells and tissues. Cell 147: 728-741, 2011.

Received November 16, 2017

Revised December 1, 2017

Accepted December 6, 2017 